\title{
Bilateral analysis of the cross-sectional area of the internal mammary arteries and veins in patients with and without breast cancer on breast magnetic resonance imaging
}

\author{
Robert-Jan Schipper • Marc B. I. Lobbes • \\ Rieky E. Dikmans • Regina G. H. Beets-Tan • \\ Marjolein L. Smidt • Carla Boetes
}

Received: 29 June 2012 / Revised: 12 December 2012 / Accepted: 18 December 2012 / Published online: 16 January 2013

(C) The Author(s) 2013. This article is published with open access at Springerlink.com

\begin{abstract}
Objective To analyse bilateral differences in the crosssectional area of the internal mammary artery (IMA) and vein (IMV) in breast cancer patients compared to healthy controls. Materials and methods On 135 breast MRIs the crosssectional areas of the IMA and IMV were measured on the
\end{abstract}

Carla Boetes is deceased.

R.-J. Schipper $(\bowtie) \cdot$ M. L. Smidt

Department of Surgery, Maastricht University Medical Center (Maastricht UMC), P.O. Box 5800, 6202 AZ Maastricht,

The Netherlands

e-mail: info@rjschipper.nl

M. L. Smidt

e-mail:m.smidt@mumc.nl

\section{R. E. Dikmans}

Department of Plastic Surgery, Maastricht University Medical Center (Maastricht UMC), P.O. Box 5800, 6202 AZ Maastricht,

The Netherlands

e-mail: rieky.dikmans@mumc.nl

R.-J. Schipper • M. B. I. Lobbes · R. G. H. Beets-Tan • C. Boetes Department of Radiology, Maastricht University Medical Center (Maastricht UMC), P.O. Box 5800, 6202 AZ Maastricht,

The Netherlands

M. B. I. Lobbes

e-mail: marc.lobbes@mumc.nl

R. G. H. Beets-Tan

e-mail: r.beets.tan@mumc.nl

M. B. I. Lobbes $\cdot$ R. G. H. Beets-Tan • M. L. Smidt • C. Boetes GROW School for Oncology and Developmental Biology, Maastricht University Medical Center (Maastricht UMC), P.O. Box 5800, 6202 AZ Maastricht, The Netherlands left and right side in the second and third intercostal space (ICS) by two independent readers. Differences were analysed using a linear mixed model.

Results In the healthy control group $(n=91)$ no significant differences between the cross-sectional areas of the IMA and IMV were observed. Both readers reported a mean adjusted difference of $0.12 \mathrm{~mm} 2(p=0.298)$ and $0.21 \mathrm{~mm} 2(p=0.058)$ for the IMA in the second ICS. In the malignancy group $(n=44)$ the cross-sectional area was significantly larger on the malignancy side compared to the contralateral side. The largest difference in the IMA was measured in the second ICS with a mean adjusted difference for reader 1 of $1.37 \mathrm{~mm} 2$ $(p<0.001)$ and for reader 2 of $0.81 \mathrm{~mm} 2(p=0.003)$.

Conclusions The vascular cross-sectional area of internal mammary vessels was significantly different on the side with breast cancer compared to the contralateral side. This difference was not observed in healthy controls.

Main messages

- MRI has become an important imaging modality in the diagnostic workup of breast cancer.

- In healthy persons no significant difference in the size of the left and right IMA is observed.

- A significant enlargement of the IMA on the malignant side occurs in most patients.

Keywords Breast Cancer · MRI · Breast vascularity · Internal mammary vessels

\section{Introduction}

Magnetic resonance imaging (MRI) has become an important imaging modality in the diagnostic workup of breast cancer patients. Breast MRI is the most accurate method for detecting breast cancer, with a reported sensitivity and specificity of 
$90 \%$ [95\% confidence interval (CI) 88-92\%] and $72 \%(95 \%$ CI $67-77 \%$ ), respectively [1]. Breast MRI could be indicated for screening in high-risk patients (i.e. BRCA mutations), in cases where multiple tumour foci are suspected, and for evaluation of the effects of neoadjuvant chemotherapy $[2,3]$. On MRI morphology, enhancement characteristics and signal intensity time curves are mostly used to differentiate between benign and malignant lesions. However, breast MRI specificity is only moderate, resulting in many false-positive findings [1]. Several studies have demonstrated a positive correlation between breast cancer and increased breast vascularity [4-8]. Nevertheless, these studies were not all able to improve the specificity of the MRI by using this information. However, a study from Schmitz et al. showed an increased specificity from $74 \%$ to $87 \%$ when adding vascular maps as a parameter, without affecting the sensitivity [9].

With respect to breast MRI, most researchers use one of the two following methods to assess changes in vascularity in the presence of malignancy. The first method focuses on prominent vessels leading to an enhanced lesion, the socalled adjacent vessel sign (AVS) [10]. The second method consists of imaging the vascularity of the whole breast on both sides with contrast-enhanced MRI [8]. However, both techniques have shortcomings.

In healthy persons the breast is mainly perfused by the internal mammary artery (IMA) [11]. It is a paired artery, one running on the left side of the sternum and one on the right side.

We hypothesised that if a malignant lesion is present the crosssectional area of the IMA and/or internal mammary veins (IMV) on the side of the lesion is increased compared to the healthy side.

Measuring and comparing the left and right IMA and IMV could only be useful if the cross-sectional areas of these vessels in a healthy person without breast malignancy are similar. However, a cadaver study of the IMA and IMV with a small population showed that the cross-sectional area of the right IMA was significantly larger than the left in healthy persons [12]. Confounders that might be responsible for this difference could probably be detected, such as anatomical variation, breast density and breast volume.

The aim of this study was to investigate whether a significant difference exists between the cross-sectional area of IMA and IMV between the healthy and the malignant side in unilateral breast cancer patients. We further investigated whether this difference is absent in healthy persons and whether the crosssectional area measurements are reproducible for various readers.

\section{Materials and methods}

\section{Patients}

In this retrospective analysis, 294 consecutive breast MRI exams were performed in 289 patients during a 3-month period. No power calculation was performed because of the proof of principle concept of this study. Exclusion criteria were history of breast-conserving surgery, mastectomy, freeflap oncoplastic reconstruction, neoadjuvant chemotherapy and patients with bilateral breast cancer. In case of multiple MRI exams of one patient, only the first MRI was included. A single radiologist independently considered all MRI exams for inclusion. A total of 125 MRI exams were excluded (Table 1). During image analysis an additional 34 MRI exams were excluded because the internal mammary artery and vein(s) were inadequately depicted, resulting in the inclusion of 135 patients. Patients were divided into two groups: a healthy control and a malignancy group. Patients with invasive breast cancer or carcinoma in situ were considered as malignant.

Imaging

MRI exams were performed at $1.5 \mathrm{~T}$ (Philips Healthcare, Best, The Netherlands) using a dedicated bilateral 16-channel breast coil. Breast MRI was performed either before or after tissue sampling. Timing and techniques used during tissue sampling were registered. For this study, a non-enhanced 3D $\mathrm{T}_{2} \mathrm{~W}$ TSE sequence was used, which is part of our standard MRI mammography protocol. The following scan parameters were used: Field of view $208 \times 369$, TR 2,000 ms, TE $205 \mathrm{~ms}$, flip angle $90^{\circ}$ in transverse plane and reconstructed voxel size $0.96 \times$ $0.96 \times 1.00 \mathrm{~mm}$. This non-enhanced sequence was used because the internal mammary vessels were best visualised on this sequence, compared to the contrast-enhanced subtraction images, which are standard in our breast MRI protocol as well. Additionally, the readers were generally blinded to the presence of breast tumours by choosing this sequence because most cancers cannot be accurately identified on nonenhanced T2-weighted images because of the large overlap of $\mathrm{T} 2$ relaxation times in benign and malignant breast lesions. It is well known that contrast enhancement is mandatory for accurate identification of breast cancers [13]. Coronal planes were reconstructed. Digital mammographies were used for the assessment of the breast density. All digital mammographies

Table 1 Reason for exclusion of patients

\begin{tabular}{lrr}
\hline Patient exclusion reason & $\mathrm{n}$ & $\%$ \\
\hline Breast-conservating therapy in history & 61 & 38 \\
Mastectomy in history & 18 & 11 \\
Free-flap oncoplastic reconstruction & 17 & 11 \\
Neoadjuvant chemotherapy & 4 & 3 \\
Bilateral breast cancer & 1 & 1 \\
Benign breast surgery in history & 5 & 3 \\
Other & 19 & 12 \\
Insufficient MRI quality & 34 & 21 \\
Total & 159 & 100 \\
\hline
\end{tabular}


were acquired on a dedicated mammography system (Senopgraphe Essential, GE Healthcare, Waukesha, WI, USA).

\section{Histopathology}

Pathological data of all included patients were gathered. All biopsies and operation specimens were classified by a pathologist according to the World Health Organisation guidelines. For this study, size and type of malignancy were gathered in the database.

Image analysis

All MRIs were assessed for regular patient care according to the BI-RADS lexicon. Additional analyses were independently performed by two readers using a dedicated viewing work station. Both readers had similar clinical experience, were not trained in reporting breast MRI and were blinded for all clinical data, including the breast MRI results. First, images were reconstructed in a coronal imaging plane by multiplanar reconstruction (Fig. 2a). Using this view the anatomical classification of the internal mammary artery and vein(s) was evaluated. It is a paired artery, one running on the left side of the sternum and one on its right side. In combination with the common running vein(s) several anatomical variations are known. The four most frequent variations were standardised by Schwabegger, the so-called Schwabegger classification [14] (Fig. 1). Discrepancies on anatomical classification were resolved by consulting a third reader, an expert reader of breast MRIs. Identification of the IMA and IMV was ensured by following the vessels to their origins. Afterwards transverse images were used. The manubrium (Fig. 2b) was used as a landmark for identifying the second rib. The cross-sectional area of the IMA and IMV(s) was measured in the second and third intercostal space (ICS) below the upper edge of the rib and a so-called "free hand region of interest" was drawn. Intraobserver reproducibility was assessed by one reader. This reader reassessed the cross-sectional area of the IMA in the second ICS in all patients with an appropriate time interval between these analyses of more than 6 months using a different coding for the separate cases to avoid recall bias. Breast density was determined on digital mammography using a semiautomatic threshold technique [15].

\section{Statistical analysis}

The interobserver agreement of anatomical variations was analysed using the unweighted Cohen's kappa coefficient. The intraobserver reproducibility was assessed by calculating the intraclass correlation coefficient (ICC) using the one-way random model. A linear mixed-model analysis was performed to investigate differences between the cross-sectional area of the left and right internal mammary vessels, accounting for the correlation between both cross-sectional area measurements within the same patient. In addition, with the use of this linear mixed model, also a possible correlation between the crosssectional area of the IMA/IMV and anatomical variation, age, breast volume or breast density was tested. In a linear mixed model, the responses from a subject are thought to be the sum (linear) of so-called fixed and random effects. If an effect affects the population mean, it is fixed. If an effect is associated with a sampling procedure, it is random. With the use of a linear-mixed model, the effects of these fixed and random effects are estimated in one model and adjustments can be made. Therefore it provides a useful approach for analysing a wide variety of data. The Statistical Package for the Social Sciences (SPSS), version 18 (Chicago, Illinois) was used for data collection and statistical analysis. P-values $<0.05$ were considered statistically significant.

\section{Results}

The median age of the control group $(n=91)$ was 50 years (range 19-79) compared to a median of 59 years (range 16$86)$ in the malignancy group $(n=44)$. The median diameter of all invasive tumours in the malignancy group was $2.20 \mathrm{~cm}$ (range $0.45-5.50 \mathrm{~cm}$ ). Table 2 shows the variation in malignancies. In the control group tissue sampling of suspicious lesions was performed in 17 patients ( 9 core biopsies, 3 stereotactic biopsies, 4 MRI-guided biopsies
Fig. 1 Four internal mammary artery and vein patterns as described by Schwabegger. Type 1 (a). Type 2 (b). Type 3 (c). Type 4 (d). From: Arnez et al., Br J Plast Surg. 1995 Dec;48(8):540-5
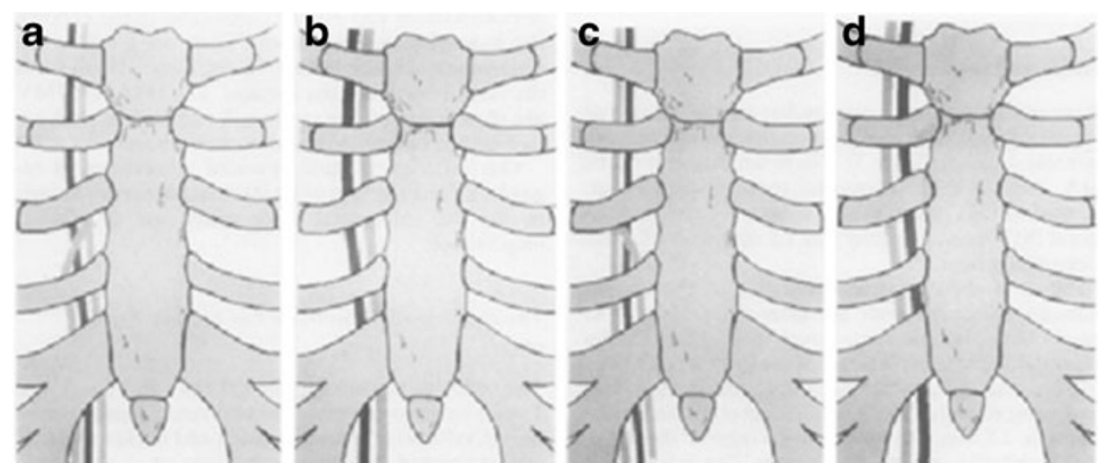

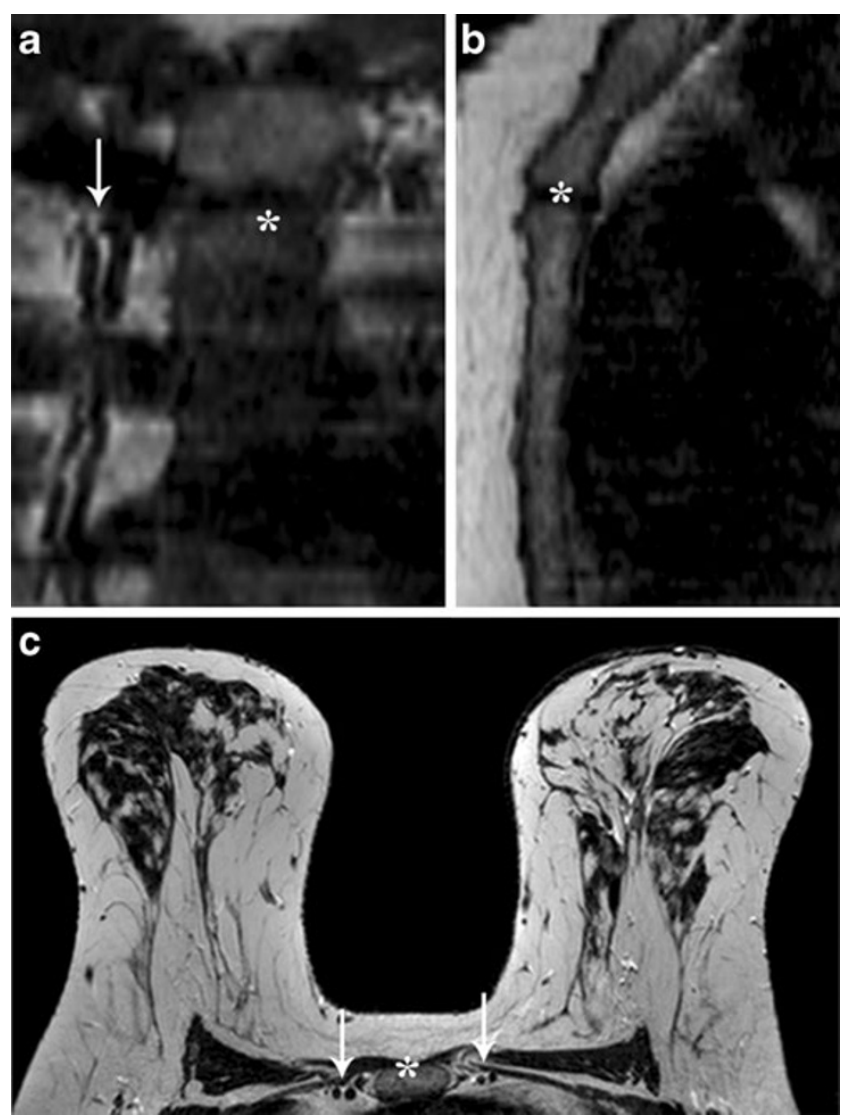

Fig. 2 An example of recognising the Schwabegger classification and measurement of the cross-sectional area of the internal mammary vessels. In this patient no malignancy was found. a Coronal view, determining the Schwabegger classification. The left internal mammary artery and vein are visible. Medial the internal mammary artery (IMA) and lateral the internal mammary vein (IMV). This anatomy is comparable with Schwabegger's type 4. b Sagittal view, marking the manubrium. This marker appears automatically in the coronal and transverse slices. c Transverse slice; using the marker the second intercostal space is recognised and the cross-sectional area of the internal mammary vessels (left and right arrows) can be measured

and 1 fine-needle aspiration cytology). In six patients biopsy was performed before breast MRI and in 11 patients afterwards. Tissue sampling was performed in all 44 patients in the malignant group [37 core biopsies, 5 stereotactic biopsies (vacuum assisted) and 2 MRI-guided biopsies]. In 35

Table 2 Type of malignant lesion

\begin{tabular}{lrr}
\hline Type of lesions & $\mathrm{n}$ & $\%$ \\
\hline Ductal carcinoma in situ & 4 & 9 \\
Invasive ductal carcinoma & 29 & 66 \\
Lobular carcinoma in situ & 1 & 2 \\
Invasive lobular carcinoma & 6 & 14 \\
Other malignancy & 4 & 9 \\
Total & 44 & 100 \\
\hline
\end{tabular}

patients breast MRI was performed after tissue sampling and in 9 patients before tissue sampling.

Classification of the anatomy of the IMA and IMV according to Schwabegger resulted in an incidence of type 1,2,3 and 4 of $16(5.9 \%), 209$ (77.4\%), $2(0.7 \%)$ and $35(13.0 \%)$ respectively. Another eight (3.0\%) anatomical variations could not be described with the Schwabegger classification [14]. For the left side, the readers' interobserver agreement showed a kappa value of 0.716 ; for the right side the kappa value was 0.728 , both indicating good agreement.

Results of cross-sectional area measurements of the IMA and IMV underneath the second and third rib are summarised in Tables 3 (control group) and 4 (malignancy group) for both readers.

Analysis of the cross-sectional area measurements performed by reader 1 of the IMA and IMV in the control group showed no significant differences between both sides in either the second or third ICS. The measured mean cross-sectional area of the IMA in the second ICS was $4.26 \mathrm{~mm}^{2}$ on the left side (range 1.99-12.41) and $4.36 \mathrm{~mm}^{2}$ on the right side (2.0811.06). The adjusted difference between the left and right IMA as result of the linear mixed model analysis was only $0.12 \mathrm{~mm}^{2}(p=0.298)$. The cross-sectional area measurement of the IMA in the second ICS was $4.32 \mathrm{~mm}^{2}$ on the left side (range 1.02-7.93) and $4.54 \mathrm{~mm}^{2}$ on the right side (range 2.158.31) measured by reader 2 . Also all other measurements of reader 2 confirmed the results of reader 1; no significant differences existed between the two sides.

In the malignancy group, the results of reader 1 showed a significant difference in the cross-sectional area of the IMA and IMV in the second as well as in the third ICS between the healthy and malignant side (see Fig. 3 for an example). The measured mean cross-sectional area of the IMA in the second ICS was $4.63 \mathrm{~mm}^{2}$ on the healthy side (range 2.0113.22 ) and $5.94 \mathrm{~mm}^{2}$ on the malignant side (2.06-13.53). For the IMA in the second ICS, the largest enlargement of an internal mammary vessel was reported with a mean adjusted difference for reader 1 of $1.37 \mathrm{~mm}^{2}(p<0.001)$. The results of reader 2 in the malignancy group showed similar results as reader 1; a significant difference existed in the second as well as in the third ICS between the healthy and malignant side, and the largest enlargement was seen in the IMA in the second ICS $\left(0.81 \mathrm{~mm}^{2}, p=0.003\right)$ too. The reproducibility of the cross-sectional area measurements of the IMA in the second ICS by reader 1 proved to be good in all patients (ICC 0.79, $95 \%$ CI 0.65-0.88).

The semi-automatic threshold technique to estimate the breast density of patients showed a median breast density of $24 \%$ with a standard deviation of $16 \%$. Together with age and anatomical variation, the mixed model analysis showed no significant correlation (all $p$ values $>0.141$ for both readers) for left-right differences of the vascular cross-sectional areas, in both the control and malignancy group. 
Table 3 Control group

\begin{tabular}{|c|c|c|c|c|c|}
\hline Control group & & $\begin{array}{l}\text { Observed mean cross-sectional } \\
\text { area }\left(\mathrm{mm}^{2}\right) \text { left side }\end{array}$ & $\begin{array}{l}\text { Observed mean cross-sectional } \\
\text { area }\left(\mathrm{mm}^{2}\right) \text { right side }\end{array}$ & Adjusted difference $\left(\mathrm{mm}^{2}\right)$ & $\mathrm{P}$ \\
\hline \multirow[t]{2}{*}{ Surface artery ICS 2} & Reader 1 & $4.26(1.99-12.41)$ & $4.36(2.08-11.06)$ & 0.12 & 0.298 \\
\hline & Reader 2 & $4.32(1.02-7.93)$ & $4.54(2.15-8.31)$ & 0.21 & 0.058 \\
\hline \multirow[t]{2}{*}{ Surface vein ICS 2} & Reader 1 & $3.35(1.88-6.74)$ & $3.51(1.97-8.01)$ & 0.19 & 0.098 \\
\hline & Reader 2 & $3.42(1.27-9.63)$ & $3.69(1.78-6.14)$ & 0.23 & 0.052 \\
\hline \multirow[t]{2}{*}{ Surface artery ICS 3} & Reader 1 & $3.66(1.88-7.22)$ & $3.78(1.93-8.88)$ & 0.11 & 0.340 \\
\hline & Reader 2 & $3.51(1.97-8.14)$ & $3.67(1.53-6.86)$ & 0.15 & 0.132 \\
\hline \multirow[t]{2}{*}{ Surface vein ICS 3} & Reader 1 & $2.92(1.48-7.73)$ & $3.13(1.57-6.88)$ & 0.20 & 0.074 \\
\hline & Reader 2 & $2.73(1.21-6.21)$ & $2.93(1.22-5.91)$ & 0.21 & 0.094 \\
\hline
\end{tabular}

In the third column the observed means are reported with the minimum and maximum for the left side. In the fourth column the same results are reported for the right side. In the fifth column the adjusted differences between left and right side vessels are reported according to the linear mixed model analysis. ICS Intercostal space

When taking a cutoff point of $3.0-\mathrm{cm}$ tumour diameter, the mean difference of the cross-sectional area in the second ICS for reader 1 was $1.43 \mathrm{~mm}^{2}$ in the group with tumours $\leq$ $3.0 \mathrm{~cm}$ compared to $1.89 \mathrm{~mm}^{2}$ in the tumours bigger $>$ $3.0 \mathrm{~cm}$. This difference was not significant.

The sensitivity was $56.4 \%(95 \%$ CI $39-72 \%)$ and specificity $85.1 \%$ (95\% CI 75-91\%) after calculating the difference between the cross-sectional area of the left and right IMA in the second ICS on a patient basis and a cutoff point of $1.00 \mathrm{~mm}^{2}$ (i.e. a difference $<1.00 \mathrm{~mm}^{2}$ between left and right, no malignancy; when $\geq 1.00 \mathrm{~mm}^{2}$, malignant). Calculated positive and negative predictive values were $62 \%$ (95\% CI $45-78 \%$ ) and $81.3 \%$ (95\% CI 71-88 \%), respectively.

\section{Discussion and further perspectives}

This study showed that in healthy persons no significant difference existed between the cross-sectional area of the left and right IMA and IMV on MRI. In the presence of malignancy this study showed a significant enlargement in the cross-sectional area of the IMA and IMV on the malignant side. With this knowledge bilateral comparison of the internal mammary vessels evaluated in the second and third ICS might be used as a predictor for the presence or absence of malignancy. Based on these results it seems most appropriate to focus on the IMA. The IMA showed the biggest enlargement of the cross-sectional area compared to the IMV. As the preferred location for the measurement of the cross-sectional area of the IMA the second ICS should be considered. The enlargement of the IMA is more explicit in the second ICS compared to the third ICS.

Increased breast vascularity can be explained by an increased demand for blood flow, providing nutrients for tumour growth [16]. In 2010 Carbognin et al. reported features of inflammatory breast cancer on MRI. They reported a hypertrophic IMA in $21 \%$ of the patients [17]. In healthy persons the breast is mainly perfused by the IMA

Table 4 Malignancy group

\begin{tabular}{|c|c|c|c|c|c|}
\hline Malignancy group & & $\begin{array}{l}\text { Observed mean cross-sectional } \\
\text { area }\left(\mathrm{mm}^{2}\right) \text { healthy side }\end{array}$ & $\begin{array}{l}\text { Observed mean cross-sectional } \\
\text { area }\left(\mathrm{mm}^{2}\right) \text { lesion side }\end{array}$ & Adjusted difference $\left(\mathrm{mm}^{2}\right)$ & $\mathrm{P}$ \\
\hline \multirow[t]{2}{*}{ Surface artery ICS 2} & Reader 1 & $4.63(2.01-13.22)$ & $5.94(2.06-13.53)$ & 1.37 & 0.000 \\
\hline & Reader 2 & $4.46(2.19-7.75)$ & $5.24(2.39-10.29)$ & 0.81 & 0.003 \\
\hline \multirow[t]{2}{*}{ Surface vein ICS 2} & Reader 1 & $3.52(0.26-7.51)$ & $4.42(1.95-13.81)$ & 0.90 & 0.005 \\
\hline & Reader 2 & $3.44(1.16-5.71)$ & $3.99(1.86-7.21)$ & 0.55 & 0.008 \\
\hline \multirow[t]{2}{*}{ Surface artery ICS 3} & Reader 1 & $3.99(2.14-10.07)$ & $5.04(1.92-12.59)$ & 1.03 & 0.000 \\
\hline & Reader 2 & $3.58(1.93-6.75)$ & $4.08(2.05-6.34)$ & 0.51 & 0.007 \\
\hline \multirow[t]{2}{*}{ Surface vein ICS 3} & Reader 1 & $3.21(1.78-7.22)$ & $3.92(1.95-7.83)$ & 0.71 & 0.002 \\
\hline & Reader 2 & $2.70(1.38-4.90)$ & $3.35(1.25-6.92)$ & 0.66 & 0.000 \\
\hline
\end{tabular}

In the third column the observed means are reported with the minimum and maximum for the side with no malignancy. In the fourth column the same results are reported for the malignant side. Column 5 shows the result of linear mixed model analysis in terms of adjusted difference in $\mathrm{mm}^{2}$. ICS Intercostal space 


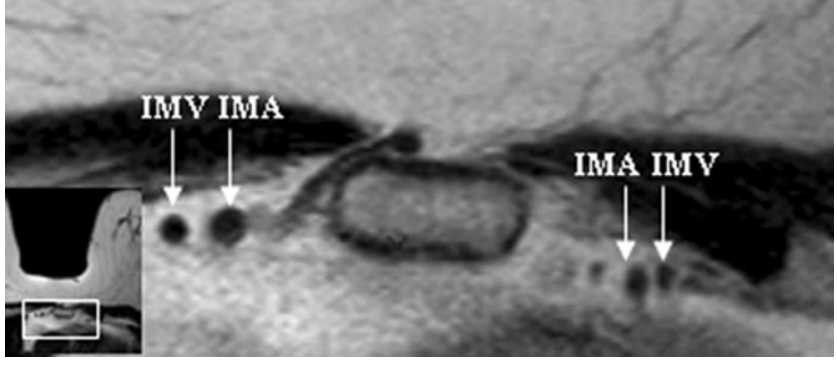

Fig. 3 An example of the internal mammary vessels in a patient with a unifocal lesion in the right breast. Measurement of the right internal mammary artery showed a cross-sectional area of $9.6 \mathrm{~mm} 2$ on the right side and $6.3 \mathrm{~mm} 2$ on the left. Histopathology showed an invasive ductal carcinoma, $3.5 \mathrm{~cm}$, grade 2, triple negative

[11]. The anatomy can vary per side in one person; therefore, comparing the left and right cross-sectional area of the IMA and IMV could be difficult. Another anatomical difference lies in the possible bifurcation of the IMV. This bifurcation occurs mostly below the third rib; using measurements only above this rib solves this problem.

Different imaging modalities were used for imaging increased vascularity, for example, laser Doppler perfusion imaging [18], positron emission tomography [19], computed tomography (CT) [20] and MRI [4-6, 8, 10, 21-23]. With respect to MRI, most researchers use one of two methods to assess changes in vascularity in the presence of malignancy. Using the AVS sign, Dietzel et al. reported a sensitivity of $47 \%$ and a specificity of $88 \%$ [10]. Others compared the vascularity of the whole breast on both sides with contrastenhanced MRI. The images were assessed according to the number of vessels, the length and maximum transverse diameter [8]. Only vessels larger than $3 \mathrm{~cm}$ in length or larger than $2 \mathrm{~mm}$ in diameter were counted. All measurements were summarised and added. A sensitivity of $88 \%$ and a specificity of $82 \%$ were reported for this method by Sardanelli et al. [8].

The two currently used methods to assess breast vascularity have different shortcomings.

Using whole breast vascularity, every branching vessel has to be considered for counting or not. This is a subjective and time-consuming procedure. Further, whole-breast vascularity assessment is not possible after mastectomy. In addition, it is possible that the diameter of the vessels increases in size, while the number of vessels stays equal [20,24].

In contrast to the whole-breast vascularity method, assessment of the AVS is possible after breast-conserving therapy (BCT) and unilateral mastectomy [24]. However, every intervention in a breast could cause an inflammation response resulting in enlargement of the (local) vessels. This can influence the sensitivity and specificity of the AVS, as Dietzel et al. already mentioned [10, 24]. They also mentioned the need to investigate the intra- and interobserver variability of the "AVS method", since assessment of the AVS is very subjective [10].
With our method a couple of the above-mentioned shortcomings can be overcome. The method is not time consuming; only two measurements of the IMA are needed. The single measurement of the IMA on the left and right side takes only $1 \mathrm{~min}$. Furthermore, the measurements in this study were reproducible by two independent readers, indicating only minor interobserver variation. However, measurement of the internal mammary vessels has its shortcomings. First of all, it is not possible in patients who have undergone a coronary artery bypass graft. After unilateral mastectomy it is also not possible to make a reliable left-right comparison of the internal mammary vessels. It is unclear whether tissue sampling in the breast influences the cross-sectional area of the internal mammary vessels because of an inflammation reaction after sampling. In this study, a case mix of patients with and without tissue sampling before breast MRI was included. We assumed that tissue sampling causes only local changes in vascularisation and does not influence the cross-sectional area of the internal mammary vessels. In one of the breast cancer patients, the MRI showed a major vascular "crossover" (see Fig. 4). A clear connection between the right and left internal mammary artery is visible. In our opinion a left-right comparison was not reliable in this case. This problem does not occur during assessment of vascularisation with the whole-breast vascularity and AVS techniques.

A limitation of this study was the fact that only the internal mammary vessels were investigated, although the breast is supplied by both the IMA and the lateral thoracic artery (LTA). A study by Doughty et al. showed that the IMA perfuses $67 \%$ of the breast and $15 \%$ of the lateral thoracic artery [11]. However, Grubstein et al. showed that the increased blood supply of the IMA or LTA was dependent on tumour localisation: $87 \%$ of the medial tumours were supplied mainly by the branches of the IMA and $13 \%$ by the branches of the LTA. The lateral tumours were mainly supplied by the IMA in $48 \%$ and by the LTA in $35 \%$. In $17 \%$ of the lateral tumours no dominant vessel

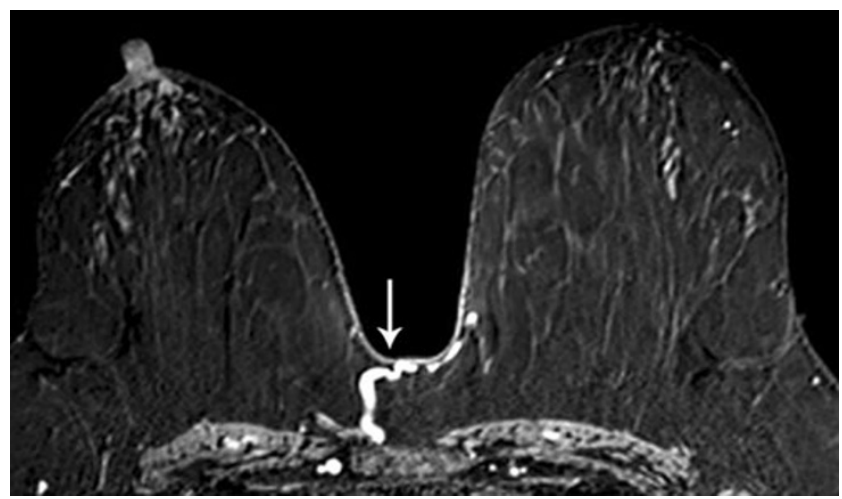

Fig. 4 Transverse view on contrast-enhanced subtraction image. A clear case of a "crossover". There is a connection between the left breast and the right internal mammary artery 
could be determined [21]. These data suggest that measurement of the IMA is not sufficient in some patients, and measurements of the LTA are needed. However, it would be very difficult to measure the LTA. Because of the anatomy of the LTA a standardised location for measurements is not available so inter- and intraobserver variability will not be as good compared to the measurements of the IMA.

Also, the visualisation of a tumour within the breast could introduce bias in the measurement, i.e. overestimation of the cross-sectional areas at the side of the breast cancer. Ideally, measurements of the vessels should be performed without visualisation of the breast itself. However, this is not possible within our viewing workstations. It is well known that tumour identification on breast MRI depends heavily on the enhancement of tissue. It is very difficult to detect breast cancers on non-enhanced T2weighted images (only larger tumours can be identified). Also, to introduce this type of bias, the tumour should be located at exactly the level of the second intercostal space to be detected while performing these measurements. We think that these considerations limit the introduction of bias in these measurements.

This study provides new insights into the possibility of using left-right IMA cross-sectional area differences as a possible new predictor for malignancy on MRI. Further research is needed on this topic. A future study should prospectively confirm these retrospective results in a larger cohort. Also the use of a dedicated MR angiographic sequence can be considered to obtain more sufficient MR quality images. In addition, we would suggest evaluating the possible correlation between tumour diameter and cross-sectional vessel enlargement or assessing the differences between various types of breast cancer, i.e. invasive versus in situ cancers. Due to the limited number of malignant cases, we were unable to perform an accurate analysis with respect to this research question. In the end, this study showed differences between both groups, which might aid in improving the specificity of breast MRI. Although this is a plausible assumption, we wish to emphasise that this is only speculative and that this theory should be evaluated in a larger prospective study, especially with the addition of this new parameter to the common used BI-RADS lexicon parameters.

Open Access This article is distributed under the terms of the Creative Commons Attribution License which permits any use, distribution, and reproduction in any medium, provided the original author(s) and the source are credited.

\section{References}

1. Peters NH, Borel Rinkes IH, Zuithoff NP, Mali WP, Moons KG, Peeters PH (2008) Meta-analysis of MR imaging in the diagnosis of breast lesions. Radiology 246(1):116-124
2. Mann RM, Kuhl CK, Kinkel K, Boetes C (2008) Breast MRI: guidelines from the European Society of Breast Imaging. Eur Radiol 18(7):1307-1318

3. Aebi S, Davidson T, Gruber G, Cardoso F (2011) Primary breast cancer: ESMO Clinical Practice Guidelines for diagnosis, treatment and follow-up. Ann Oncol 22(Suppl 6):vi12-vi24

4. Siewert C, Oellinger H, Sherif HK, Blohmer JU, Hadijuana J, Felix R (1997) Is there a correlation in breast carcinomas between tumor size and number of tumor vessels detected by gadoliniumenhanced magnetic resonance mammography? MAGMA 5(1):2931

5. Ando Y, Fukatsu H, Ishiguchi T, Ishigaki T, Endo T, Miyazaki M (2000) Diagnostic utility of tumor vascularity on magnetic resonance imaging of the breast. Magn Reson Imaging 18(7):807-813

6. Kul S, Cansu A, Alhan E, Dinc H, Reis A, Can G (2010) Contrastenhanced MR angiography of the breast: evaluation of ipsilateral increased vascularity and adjacent vessel sign in the characterization of breast lesions. AJR Am J Roentgenol 195(5):1250-1254

7. Sardanelli F, Fausto A, Menicagli L, Esseridou A (2007) Breast vascular mapping obtained with contrast-enhanced MR imaging: implications for cancer diagnosis, treatment, and risk stratification. Eur Radiol 17(Suppl 6):F48-F51

8. Sardanelli F, Iozzelli A, Fausto A, Carriero A, Kirchin MA (2005) Gadobenate dimeglumine-enhanced MR imaging breast vascular maps: association between invasive cancer and ipsilateral increased vascularity. Radiology 235(3):791-797

9. Schmitz AC, Peters NH, Veldhuis WB et al (2008) Contrastenhanced 3.0-T breast MRI for characterization of breast lesions: increased specificity by using vascular maps. Eur Radiol 18 (2):355-364

10. Dietzel M, Baltzer PA, Vag T et al (2010) The adjacent vessel sign on breast MRI: new data and a subgroup analysis for 1,084 histologically verified cases. Korean J Radiol 11(2):178-186

11. Doughty JC, McCarter DH, Kane E, Reid AW, Cooke TG, McArdle CS (1996) Anatomical basis of intra-arterial chemotherapy for patients with locally advanced breast cancer. Br J Surg 83(8): $1128-1130$

12. Feng LJ (1997) Recipient vessels in free-flap breast reconstruction: a study of the internal mammary and thoracodorsal vessels. Plast Reconstr Surg 99(2):405-416

13. Heywang SH, Wolf A, Pruss E, Hilbertz T, Eiermann W, Permanetter W (1989) MR imaging of the breast with Gd-DTPA: use and limitations. Radiology 171(1):95-103

14. Schwabegger AH, Ninkovic MM, Moriggl B et al (1997) Internal mammary veins: classification and surgical use in free-tissue transfer. J Reconstr Microsurg 13(1):17-23

15. Lobbes MB, Cleutjens JP, Lima Passos V et al (2012) Density is in the eye of the beholder: visual versus semi-automated assessment of breast density on standard mammograms. Insights Imaging 3 (1):91-99

16. Weidner N, Folkman J, Pozza F et al (1992) Tumor angiogenesis: a new significant and independent prognostic indicator in early-stage breast carcinoma. J Natl Cancer Inst 84(24):1875-1887

17. Carbognin G, Calciolari C, Girardi V, Camera L, Pollini G, Pozzi Mucelli R (2010) Inflammatory breast cancer: MR imaging findings. Radiol Med 115(1):70-82

18. Seifalian AM, Chaloupka K, Parbhoo SP (1995) Laser Doppler perfusion imaging-a new technique for measuring breast skin blood flow. Int J Microcirc Clin Exp 15(3):125-130

19. Wilson CB, Lammertsma AA, McKenzie CG, Sikora K, Jones T (1992) Measurements of blood flow and exchanging water space in breast tumors using positron emission tomography: a rapid and noninvasive dynamic method. Cancer Res 52(6):1592-1597

20. Kang DK, Kim EJ, Kim HS, Sun JS, Jung YS (2008) Correlation of whole-breast vascularity with ipsilateral breast cancers using contrast-enhanced MDCT. AJR Am J Roentgenol 190(2):496-504 
21. Grubstein A, Yepes M, Kiszonas R (2010) Magnetic resonance imaging of breast vascularity in medial versus lateral breast cancer. Eur J Radiol 75(2):e9-e11

22. Mahfouz AE, Sherif H, Saad A et al (2001) Gadoliniumenhanced MR angiography of the breast: is breast cancer associated with ipsilateral higher vascularity? Eur Radiol 11 (6):965-969
23. Wright H, Listinsky J, Quinn C, Rim A, Crowe J, Kim J (2005) Increased ipsilateral whole breast vascularity as measured by contrast-enhanced magnetic resonance imaging in patients with breast cancer. Am J Surg 190(4):576-579

24. Sardanelli F (2010) Vessel analysis on contrast-enhanced MRI of the breast: global or local vascularity? AJR Am J Roentgenol 195 (5):1246-1249 